\title{
Dismorfia Muscular: detección del uso-abuso de esteroides anabolizantes androgénicos en una muestra española
}

\section{Muscle dysmorphia: detection of the use-abuse of anabolic androgenic steroids in a Spanish sample}

\author{
Irene González-Martí*, Juan Gregorio Fernández-Bustos**, Onofre Ricardo \\ Contreras Jordán**, Marina SOKOLOVA***.
}

*Universidad de Castilla-La Mancha. Facultad de Educación. Departamento de Educación Física. Edificio Fray Luis de León, Avenida de los Alfares 42, 16071, Cuenca, España; **Universidad de Castilla-La Mancha, Facultad de Educación. Departamento de Educación Física. Edificio Simón Abril, Plaza de la Universidad 3, 02071, Albacete, España; ***Southwest State University, Kursk, Rusia.

\section{Resumen}

Las personas que padecen Dismorfia Muscular (DM) debido a una distorsión en la imagen corporal, se perciben menos musculosas de lo que son en realidad. Para paliar este problema y con el fin de aumentar su musculatura, algunas de estas personas hacen uso de hormonas ilegales, como son los esteroides anabolizantes androgénicos (EAA), cuya función principal es aumentar la musculatura. El objetivo de este estudio es conocer la prevalencia del uso de EAA en personas afectadas por Dismorfia Muscular. La muestra de este estudio estaba compuesta de 562 hombres y 172 mujeres fisicoculturistas y levantadores de pesas, a los que se le administraron medidas antropométricas, la ecuación Fat-Free Mass Index, el cuestionario Escala de Satisfacción Muscular y el test informatizado Somatomorphic Matrix. Como resultado se crearon diferentes modelos de regresión de la DM, empleando las técnicas estadísticas de árboles de decisión $(R=.78 \mathrm{y}$ $R 2=.62)$ de minería de datos. La principal conclusión es que el $50 \%$ de participantes afectados por este trastorno usa EAA.

Palabras clave: dismorfia muscular; esteroides; fisicoculturistas.

\begin{abstract}
People who suffer from muscle dysmorphia due to a distorted body image perceive themselves as less muscular than they actually are. With the aim of increasing their muscular development, they resort to the use of anabolic androgenic steroids (AAS). The purpose of this study is to know the prevalence of the use of AAS in a Spanish sample affected by muscle dysmorphia. The study sample was comprised of 562 male and 172 female bodybuilders and weightlifters who were applied anthropometric measurements, Fat-Free Mass Index equation, Escala de Satisfacción Muscular, and Somatomorphic Matrix software. The results show the creation of decision trees and a regression model was used to create explanatory models for muscle dysmorphia $(R=.78$ and $R 2=.62$ ). The main conclusion is that almost $50 \%$ of both male and female participants affected by this disorder use this kind of AAS. Key words: muscle dysmorphia; steroids; bodybuilders.
\end{abstract}




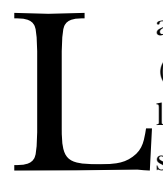

as personas que padecen Dismorfia Muscular (DM) sufren de un trastorno mental que conlleva una imagen corporal distorsionada por subestimación del tamaño muscular (Pope, Phillips, y Olivardia, 2000). Hace poco, este trastorno se ha incluido en el DSM-V como Trastorno Obsesivo-compulsivo (Rodríguez-Testal, Senín-Calderón, y Perona-Garcelán, 2014). Consiguientemente, una de las características más comunes de este trastorno es el entrenamiento obsesivo con pesas con el propósito de mejorar la percepción de la apariencia física debido a los cambios que esta práctica produce sobre el cuerpo. Por esta razón, los fisioculturistas y los levantadores de pesas son el grupo de deportistas más afectados por este trastorno, ya que mediante el deporte hallan una solución para incrementar su musculatura (Barrientos, Escoto, Bosques, Enríquez, y Juárez, 2014; González-Martí, 2012).

No obstante, el aumento de su musculatura no resulta únicamente de su entrenamiento agotador. Existen métodos artificiales que aumenta el tamaño de sus músculos de manera más rápida y con menos esfuerzo. Estos métodos artificiales se basan en el uso de esteroides anabolizantes androgénicos (EAA) (Kanayama, Barry, Hudson, y Pope, 2006). Son "sustancias de la imagen corporal", como los llaman Kanayama et al. (2006), porque no se usan por su acción psicoactiva, sino por su efecto sobre la imagen corporal (Coomber et al., 2014; Van Hout y Kean, 2015) por parte de las personas afectadas por DM (Rohman, 2009). Por sus efectos secundarios nocivos para la salud del deportista, estas sustancias exógenas son ilegales en el deporte sin receta médica. No obstante, el deseo de tener musculatura se convierte en un fin en sí mismo, y el deportista cae en la tentación de usar sustancias ilegales para mejorar su rendimiento y su nivel muscular (Rocha, Aguiar, y Ramos, 2014). Además, los usuarios de estas sustancias puede aumentar la intensidad y la frecuencia de los entrenamientos porque la recuperación entre uno y otro es más rápida.

Estas sustancias pueden ser detectadas usando métodos directos e indirectos. De los primeros, los más comunes son las analíticas de orina y/o sangre, mientras que los indicadores de los segundos derivan de la ecuación del Fat-Free Mass Index (FFMI; Kouri, Pope, Katz, y Oliva, 1995) que ofrece mayor información sobre el nivel de la masa muscular magra, y si el mismo se ha conseguido usando EAA.

Las sustancias para mejorar el rendimiento más solicitadas por personas afectadas por DM son hormonas del crecimiento y testosterona. La testosterona es un esteroide natural sintetizado por las células intersticiales en los testículos en los hombres y por los ovarios y el córtex adrenal en las mujeres (De la Torre, 1995). Su función androgénica estimula las características sexuales masculinas, como la aparición del lanugo y la producción de la voz grave y viril por la hipertrofia de la laringe. El efecto anabólico aumen- ta la musculatura. El hombre produce entre 5-10 $\mathrm{mg}$ de testosterona diaria. La concentración de testosterona en un hombre adulto sano es entre 12 y $28 \mathrm{nmol} /$, y en una mujer es el $5 \%$ de la concentración del hombre (De la Torre, 1995). El hipogonadismo es un trastorno hormonal en el cual la persona no produce la cantidad de testosterona que corresponde a un individuo sano. Un estudio de Bhasin et al. (1997) demuestra que si la administración de testosterona a pacientes con hipogonadismo aumenta su peso corporal muscular en un 6\%, y su Fat-Free Mass Index en un $9 \%$, el uso de testosterona en personas sanas duplicaría dichos porcentajes. Por ello, deja de ser sorprendente que las personas afectadas por DM usen esta hormona de manera exógena en su afán de aumentar su musculatura (Pipet, Halpern, Woody, y Szobot, 2014).

La testosterona puede ser administrada por vía oral o vía inyectada porque es biodegradable por el organismo (De la Torre, 1995). Por ello, su estructura química puede ser sometida a cambios en el laboratorio para producir EAA. Los EAA son las hormonas más frecuentemente usadas por fisioculturistas y levantadores de pesas para aumentar su musculatura y reducir su grasa corporal (Jampel, Murray, Griffiths, y Blashill, 2016).

No hay estudios en España sobre el impacto de los EAA en personas afectadas por DM, y existe una asociación entre las consecuencias negativas para la salud del uso y abuso de las EAA y los riesgos psiquiátricos, hepáticos, endocrinos, neurológicos, cardiovasculares, y de mortalidad (Pope et al., 2014, Thiblin et al., 2015) y con un volumen cerebral y grosor cortical menores (Bjørnebekk et al., 2017). Por ello, el objetivo de este estudio es definir la prevalencia del uso de los EAA en hombres y mujeres españoles, fisioculturistas y levantadores de pesas, afectados por DM, usando métodos indirectos del Fat-Free Mass Index. Partimos de la hipótesis que el uso de estas hormonas aumentadoras de la musculatura en los participantes con este trastorno será alta, dado que su distorsión y su deseo de ganar tamaño y fuerza les impulsa a usar estas sustancias ilegales.

\section{Método}

\section{Participantes}

La muestra estaba integrada por 734 fisioculturistas y levantadores de pesas, 562 hombres y 172 mujeres. Los hombres tenían edades entre los 16 - 63 años $(M=29,38$, $D T=8,45)$, mientras que las mujeres comprendidas tenían edades entre los 16 - 61 años $(M=32,07, S D=9,04)$. La altura media de los hombres fue de $1,74 \mathrm{~m}$. $(S D=6,63)$, y de las mujeres $1,61 \mathrm{~m}$. $(S D=5,82)$. El peso medio de los hombres era 78,06 kg $(S D=10,46)$, y de las mujeres 59,58 $\mathrm{kg}(S D=8,34)$. Según autoinformes de los participantes, los hombres y las mujeres dedicaban semanalmente 8,22 horas $(S D=3,58)$ y 6,56 horas $(S D=4,15)$, respectivamente, al entrenamiento con pesas y al ejercicio aeróbico. 
Tabla 1. Instrumentos aplicados para detectar DM

\begin{tabular}{ll}
\hline Instrumentos & Variables/factores y coeficiente de fiabilidad \\
\hline Cuestionario Demográfico & Sexo, uso de suplementos y/u hormonas, y prácticas deportivas \\
$\begin{array}{l}\text { Escala de Satisfacción Muscular } \\
\text { (ESM; González-Martí, Fernández, Contreras, y Mayville, 2012) }\end{array}$ & Dependencia de Fisioculturismo, Uso de Sustancias, Lesión, \\
$\begin{array}{l}\text { Somatomorphic Matrix } \\
\text { (SM; Gruber, Pope, Borowiecki, y Cohane, 1999) }\end{array}$ & FFMI percibido y deseado, y Grasa Corporal percibida y deseada.
\end{tabular}

Tabla 2. Categorías del FFMI, según sexo

\begin{tabular}{llll}
\hline Categoría & Hombres $\left(\mathrm{kg} / \mathrm{m}^{2}\right)$ & Mujeres $\left(\mathrm{kg} / \mathrm{m}^{2}\right)$ & Nivel de musculatura \\
\hline 1 & $16,5-19,9$ & $11,5-14,9$ & Leve \\
2 & $20-22,9$ & $15-17,9$ & Perceptible \\
3 & $23-24,9$ & $18-19,9$ & Notable \\
4 & $25-25,9$ & $20-20,9$ & Muscular (uso probable de EAA) \\
5 & $\geq 26$ & $\geq 21$ & Muy muscular (resultado de uso-abuso de AAS) \\
\hline
\end{tabular}

\section{Instrumentos}

Para detectar dismorfia muscular. Se usaron cuestionarios abreviados cuyas respuestas fueron evaluadas por un grupo de expertos para clasificar a una persona con trastorno de DM. Dichos instrumentos se exponen en la Tabla 1.

Para detectar el uso de EAA. Dentro del cuestionario demográfico sobre el uso de estas sustancias, se incluyó un grupo de preguntas directas con tres respuestas posibles: nunca, a veces y siempre. También se preguntó acerca de la razón por la cual el participante usa la hormona, su función y una pregunta abierta sobre el tipo de hormona usada. Se administró la ecuación del Fat-Free Mass Index (FFMI) diseñada por Kouri et al. (1995) para conocer el nivel de musculatura de cada participante y si dicha musculatura se alcanzó usando esteroides. Con este objetivo, y según el protocolo estandarizado de la Sociedad Internacional para el Avance de la Cineantropometría (ISAK), se tomaron medidas antropométricas de seis pliegues cutáneos en los hombres y tres en las mujeres. Estas medidas, con la edad del participante, se aplican a la ecuación creada por Jackson y Pollock (1978) para obtener el porcentaje de grasa corporal. Los datos resultantes se introducen en la ecuación del FFMI, que varía para cada sexo. La ecuación para las mujeres es: [(peso (100-\% grasa corporal) /100) altura $^{2}$ ], y para los hombres: [ [ (peso (100-\% grasa corporal) 100) altura $\left.{ }^{2}\right]+6,1(1,8$ - altura) $]$. La altura se mide en metros y el peso en kilos. Se establecieron unas categorías para saber si la masa muscular obtenida con la ecuación del FFMI resultó del uso de esteroides. Están basadas en las categorías propuestas por Kouri et al. (1995), Pope et al. (2000) y Kyle, Schutz, Dupertuis, y Pichard (2003). La Tabla 2 muestra las categorías del FFMI usadas en este estudio.

\section{Procedimiento}

Para la administración de los instrumentos. Después de los contactos iniciales, nos dirigimos a los gerentes de gimnasios para obtener la autorización para realizar este estudio en sus instalaciones deportivas. Tras explicar el propósito del estudio, algunos de los gimnasios en España optaron por no participar en el mismo por motivos de detección del uso de EAA. Finalmente, participaron trece gimnasios de las provincias españolas de Albacete, Murcia, Valencia y Madrid. Una vez preparados los recursos necesarios en un espacio dispuesto para este fin, se explicó a los participantes con un notable físico muscular el objetivo del estudio, el anonimato de los resultados, y la voluntariedad de su participación. Si estaban de acuerdo, firmaban un documento de consentimiento informado. Los participantes menores de edad acudieron con su padre/madre o tutor legal para que autorizase su participación en el estudio, y ambas partes firmaron el documento de consentimiento informado. El procedimiento nunca duraba más de veinticinco minutos. El Comité de Ética de la Universidad de Castilla-La Mancha aprobó el estudio.

Para detectar si el participante padece DM. Para detectar patrones ocultos e investigar qué factores tienen mayor impacto sobre la DM, se usaron árboles de decisiones entrenados con algoritmos M5P y REPTree. Además, se crearon modelos diferentes explicativos de DM usando modelos de análisis de regresión (González-Martí, 2012; González-Martí, Fernández, y Contreras, 2012; Sokolova, González-Martí, Contreras, y Fernández, 2013) y se seleccionó el que mejor explicaba este trastorno con valores más altos, $R=.786$ y $R 2=.62$, lo que significa que los variables explicativas describían el $62 \%$ del variable clase.

Dependencia de Fisioculturismo $=-0.016$ Edad -0.027 Grasa Corporal Deseada +0.466 Conductas de verificación +0.281 Uso 
Tabla 3. Clasificación de DM según las categorías de ESM y SM

\begin{tabular}{|c|c|c|c|c|}
\hline Instrumento & Puntuación & Categoría & Categorización & DM \\
\hline \multirow{4}{*}{$\begin{array}{l}\text { ESM } \\
\text { (González-Martí et al., } \\
\text { 2014) }\end{array}$} & $19-38$ & 1 & Satisfacción Muscular & No \\
\hline & $39-57$ & 2 & Insatisfacción Muscular Leve & No \\
\hline & $58-76$ & 3 & Insatisfacción Muscular Moderada & Sí \\
\hline & 77 & 4 & Insatisfacción Muscular Severa & Sí \\
\hline Instrumento & Discrepancia entre actual, percibido e ideal & Categoría & Categorización & DM \\
\hline \multirow{4}{*}{$\begin{array}{l}\text { SM } \\
\text { (Pope et al., 2000) }\end{array}$} & Grasa corporal de $1-15 \%$ & 1 & Ninguna distorsión de imagen corporal & No \\
\hline & Grasa corporal >15\% & 2 & $\begin{array}{l}\text { Imagen corporal distorsionada, como Anorexia o } \\
\text { DM }\end{array}$ & Sí \\
\hline & FFMI de $0-2,9 \mathrm{~kg} / \mathrm{m}^{2}$ & 1 & Ninguna distorsión de imagen corporal & No \\
\hline & $\mathrm{FFMI} \geq 3 \mathrm{~kg} / \mathrm{m}^{2}$ & 2 & Imagen corporal distorsionada, DM & Sí \\
\hline
\end{tabular}

de Sustancias +0.467 Lesión -0.059 Atractivo Físico +0.092

Fuerza- 0.032 Auto-concepto físico general +3.714 .

Los atributos del modelo se seleccionaron con la ayuda del cuestionario Escala de Satisfacción Muscular (ESM: González-Martí et al., 2012) y sus categorías (González-Martí, Fernández, Hernández-Martínez, y Contreras, 2014) y las de la Somatomorphic Matrix (SM; Gruber et al.; 1999; Pope et al., 2000). Dichas categorías ofrecen la información necesaria para saber si un paciente padece, o no, este trastorno, según las respuestas obtenidas del cuestionario y de los modelos de clasificación. La Tabla 3 muestra la probabilidad de tener DM en relación a estas categorías.

Por lo tanto, se consideró que un participante tenía trastorno de DM si sus resultados se clasificaban en las categorías 3 o 4 de la ESM (González-Martí, 2012; González-Martí et al., 2014) y en la categoría 2 de grasa corporal y/o en la categoría 2 del FFMI de la MS (Pope et al., 2000).

\section{Resultados}

\section{Categorías del FFMI actual,}

basado en la declaración de haber usado EAA

El $32 \%$ de los hombres participantes que admitió su uso ocasional de EAA para aumentar su musculatura pertenece a la categoría 5 del FFMI; dicho de otra manera, su musculatura resulta del uso de estas sustancias, también el $21,4 \%$ de los hombres con un uso continuo de EAA. No obstante, el 3,1\% de los hombres participantes declaró no haber usado nunca sustancias ilegales sin receta médica, lo contrario a lo que muestran sus FFMI. Respecto a las mujeres, todas las participantes en este estudio negaron el uso de EAA, mientras que, según sus FFMI (Kouri et al., 1995), el $11 \%$ usó estas sustancias y el 4,7\% podría estar usándolas, al pertenecer a las categorías 5 y 4 del FFMI, respectivamente (Kouri et al., 1995; Pope et al., 2000).

E1 $100 \%$ de la muestra que declaró su uso de este tipo de hormonas lo hizo con el propósito de aumentar su musculatura. Los EAA (Hildebrandt et al., 2011) más usados por los hombres que admitieron su uso ocasional o continuo fueron Deca-Durabolin (78\%) y Dianabol (22\%), y los más usados por las mujeres eran Androgel (47\%), Dianabol $(33 \%)$ y Deca-Durabolin $(20 \%)$.

\section{Categorías del FFMI, según sexo}

Realizamos un análisis de frecuencia para detectar la distribución de los participantes, según las categorías establecidas. De esta manera, supimos que el 5,9\% de los hombres tenía un físico muscular que provocaba sospecha del uso de EAA (categoría 4), mientras que el 4,8\% de los hombres tenía una masa muscular magra que sobrepasaba $26 \mathrm{~kg} / \mathrm{m}^{2}$, resultado del uso de EAA. El 4,7\% de las mujeres tenía una musculatura muy desarrollada, muy difícilmente alcanzable de forma natural (categoría 4), y el 11\% tenía un FFMI elevado como consecuencia del uso de EAA (categoría 5) (Kouri et al., 1995; Pope et al., 2000).

Detección del uso de EAA en participantes afectados por DM

La detección de DM hallada en este estudio, según la clasificación de la Tabla 3 basada en el árbol de decisiones de la minería de datos, es de $18,3 \%(N=734)$. Del total

Tabla 4. Detección del uso de EAA en participantes afectados por DM

\begin{tabular}{llll}
\hline Categorías FFMI & DM & \multicolumn{1}{c}{$\begin{array}{c}\text { Sexo } \\
\text { Hombres } \\
\text { Frecuencia (\%) }\end{array}$} & $\begin{array}{l}\text { Mujeres } \\
\text { Frecuencia (\%) }\end{array}$ \\
\hline 1 & No & $76(90,5)$ & $11(100)$ \\
& Sí & $8(9,5)$ & $\cdots--$ \\
\hline 3 & No & $252(87,5)$ & $91(84,3)$ \\
& Sí & $36(12,5)$ & $17(15,7)$ \\
\hline 4 & No & $102(78,5)$ & $16(61,5)$ \\
& Sí & $28(21,5)$ & $10(38,5)$ \\
\hline 5 & No & $23(69,7)$ & $4(50)$ \\
& Sí & $10(30,3)$ & $4(50)$ \\
\hline
\end{tabular}

Nota. Categorías FFMI para hombres: $1=16,5-19,9 \mathrm{~kg} / \mathrm{m}^{2} ; 2=20-22,9 \mathrm{~kg} / \mathrm{m}^{2}$; $3=23-24,9 \mathrm{~kg} / \mathrm{m}^{2} ; 4=25-25,9 \mathrm{~kg} / \mathrm{m}^{2} ; 5=\geq 26 \mathrm{~kg} / \mathrm{m}^{2}$; Categorías FFMI para mujeres: $1=11,5-14,9 \mathrm{~kg} / \mathrm{m}^{2} ; 2=15-17,9 \mathrm{~kg} / \mathrm{m}^{2} ; 3=18-19,9 \mathrm{~kg} / \mathrm{m}^{2} ; 4=20-20,9$ $\mathrm{kg} / \mathrm{m}^{2} ; 5=\geq 21 \mathrm{~kg} / \mathrm{m}^{2}$. 
afectado por este trastorno $(N=134)$, el $70,1 \%$ corresponde al grupo de hombres $(n=94)$, mientras que el $29,9 \%$ representaba al grupo de mujeres $(n=40)$. El $44.4 \%$ de los hombres tenía DM y usaba EAA, mientras que se sospechaba que el 30,3\% de los hombres participantes usaban estas sustancias, como muestra la Tabla 4. El 47,4\% de las mujeres tenía DM y usaba EAA, mientras que se sospechaba que el $50 \%$ de las mujeres participantes usaba estas sustancias ilegales.

Como muestra la Tabla 5, considerando únicamente a esos deportistas clasificados con DM, tras aplicar el análisis multivariante de las diferentes variables estudiadas (escalas ESM y FFMI actual-FFMI percibido), vemos como las categorías 4 y 5 del FFMI, es decir, las personas cuyo índice de masa muscular muestra el uso de EAA para aumentar la musculatura, y las que tienen una musculatura que provocaba sospecha del uso de EAA, componen el grupo más afectado por DM, en comparación con otros con un FFMI más discreto (categorías 1, 2, y 3) y obtuvieron puntuaciones significativamente más altas $(p<.05-.001)$ en todas las variables comparadas.

\section{Discusión}

El propósito de este estudio fue detectar el uso de EAA en la muestra española afectada por DM, dada la inexistencia de estudios actuales que establezcan dicha relación en este contexto cultural. Con este objetivo, desglosamos los resultados por sexo, debido a que así lo requiere el uso del instrumento FFMI aplicado como método indirecto para detectar el uso y abuso de EAA. Es más, se les preguntó directamente a los participantes si usaban hormonas, o no, y con qué frecuencia. El uso de estas sustancias ilegales se inicia con el fin de aumentar la masa y calidad muscular y de perder grasa, un hecho directamente relacionado con el trastorno de DM (Hildebrandt, Harty, y Langenbucher, 2012; Kanayama, Pope, Cohane, y Hudson, 2003; Kana- yama et al., 2006; Kanayama, Hudson, y Pope, 2009; Olivardia, Pope, y Hudson, 2000; Pope et al., 2005). Por este motivo, la relación entre el uso de EAA y DM es bidireccional, de tal forma que el uso de esteroides ha sido aceptado como indicador de aumento de musculatura y DM (Kanayama et al., 2006; Murray, Griffiths, Mond, Kean, y Blashill, en prensa). También, el uso de estas drogas aumenta por parte de las personas con este trastorno (Pope et al. 2005).

El 21,4\% de los hombres participantes en el estudio corroboró su uso de EAA a través de sus respuestas a la pregunta sobre la frecuencia de uso. De todas maneras, esta cifra podría ser mayor, dado que el FFMI del 3,1\% de los participantes mostró que eran usuarios de EAA, aunque no lo confirmen. Ninguna de las mujeres declaró el uso de EAA, pero su FFMI de 15,7\% muestra que sí los usan. Estos resultados pueden deberse a la ilegalidad del uso de esteroides en el deporte sin receta médica. Es difícil establecer la prevalencia exacta de uso porque las personas que las usan lo hacen de manera clandestina, la mayoría de las veces sin receta médica, y adquiriendo los EAA en el mercado negro (Coomber et al., 2014; Pope et al., 2000; Van Hout y Kean, 2015).

Según los resultados hallados por el uso del FFMI como método indirecto para conocer el uso de esteroides por los participantes, las mujeres en este estudio ofrecieron datos similares a los de los hombres con respecto del porcentaje de la muestra de la cual se sospecha el uso de EAA según el FFMI (4,7\% y 5,9\%, respectivamente), y resultados aún mayores en la categoría 5 , es decir, en el caso de haber sido usuarios de EAA ( $11 \%$ de mujeres y $4,8 \%$ de hombres).

Consiguientemente, hay una correlación elevada entre el uso de EAA y el nivel de musculatura de una persona y, también, el trastorno de DM. Las respuestas a las preguntas abiertas mostraron que los participantes que admitieron el uso de EAA de forma continua, tanto como los que admitieron el uso de EAA de forma ocasional, tenían un FFMI actual más alto que los participantes que no usaban EAA.

Tabla 5. Resultados descriptivos y MANOVA de ESM y FFMI actual-percibido según la inclusión de categorías FFMI basadas en el posible uso-abuso o no de EAA

\begin{tabular}{|c|c|c|c|c|c|c|}
\hline \multirow[t]{2}{*}{ Variables } & $\begin{array}{c}\Sigma \text { Categorías } \\
\text { FFMI } 1,2,3 \\
\text { (no-uso de EAA) } \\
\quad(N=99)\end{array}$ & $\begin{array}{c}\Sigma \text { Categorías } \\
\text { FFMI 4,5 (posible } \\
\text { uso de EAA) } \\
(N=35)\end{array}$ & MANOVA & & & \\
\hline & $M$ & $S D$ & $M$ & $S D$ & $F$ & $p$ \\
\hline Dependencia de Fisioculturismo & 11,46 & 4,43 & 14,69 & 6,42 & 10,64 & $.001\left(^{\star \star}\right)$ \\
\hline Conductas de verificación & 7,95 & 3,93 & 10,91 & 5,54 & 11,71 & $.001\left(^{\star \star}\right)$ \\
\hline Uso de sustancias & 6,86 & 3,70 & 10,17 & 5,64 & 15,43 & $.000\left(^{\star \star \star}\right)$ \\
\hline Lesión & 6,76 & 3,03 & 8,74 & 3,57 & 10,07 & $.002\left(^{\star \star}\right)$ \\
\hline Insatisfacción Muscular & 10,06 & 2,68 & 11,37 & 2,57 & 6,28 & $.013\left(^{\star}\right)$ \\
\hline FFMI actual-percibido & 3,01 & 2,72 & 7,35 & 4,17 & 48,71 & $.000(\star \star \star)$ \\
\hline
\end{tabular}

Nota. ${ }^{\star} p<.05 ;{ }^{\star \star} p<.01 ;{ }^{* \star *} p<.001$. 
Respecto a esto, Hildebrandt, Schlundt, Langenbucher, y Chung (2006) obtuvieron, en una muestra de hombres con DM, un IMC medio de $27,46 \mathrm{~kg} / \mathrm{m}^{2}$, correspondiente a $24,5 \mathrm{~kg} / \mathrm{m}^{2}$ según el FFMI, lo que reveló el uso de EAA para obtener este nivel superior de masa muscular magra. De manera similar, Kanayama et al. (2006) halló en un estudio realizado con levantadores de pesas que la imagen corporal distorsionada asociada con DM estaba directamente relacionada con el uso de EAA.

En este sentido, Kanayama et al., (2009) y Cole, Smith, Halford, y Wagstaff (2003) encontraron una correlación fuerte entre los usuarios dependientes de EAA y el trastorno de MD. Esta correlación fue de nuevo corroborada por Pope, Kanayama, y Hudson (2012), en su estudio en el cual los levantadores de pesas con DM también eran usuarios de EAA. Pope et al., (2005) concluyeron que la gran mayoría de participantes con DM usaban esteroides para aumentar su masa muscular (Pope, Kanayama, Ionescu-Pioggia, y Hudson, 2004; Pope et al., 2005), y que también tenían una tasa elevada de uso de otras drogas.

Este estudio detectó el uso de EAA sw la muestra afectada por DM en el 44,4\% de los hombres y en el 47,4\% de las mujeres, dentro de la categoría 5 del FFMI. Estos resultados pueden ser aún mayores si incluimos los participantes cuyo nivel de FFMI es sospechoso del uso de este tipo de drogas, más los participantes que actualmente usan dichas drogas pero cuyo FFMI todavía no lo ha revelado.

Como se puede ver, la detección del uso de esteroides por los participantes en este estudio es ligeramente más alto en las mujeres que en los hombres. Este hecho puede discrepar con la literatura existente, en el cual el porcentaje de hombres que usa EAA es más alto (González-Martí, 2012). Según estos autores, tres millones de deportistas son usuarios de estas sustancias, el $90 \%$ de fisioculturistas hombres y el $80 \%$ de fisioculturistas mujeres.

El hecho de que este estudio tenga una población de mujeres más grande que los estudios previos sobre DM y el uso de esteroides, puede explicar la detección superior del uso de EAA en la población femenina (González-Martí, Hernández-Martínez, Fernández, \& Contreras, 2016). Los únicos resultados comparables en cuanto a la prevalencia del uso de esteroides en mujeres participantes son los de Pope, Gruber, Choi, Olivardia, y Phillips (1997), en los cuales el 38\% de mujeres fisioculturistas usaba este tipo de droga, una cifra menor que la nuestra. Respecto de los hombres, obtuvimos resultados similares a los de Olivardia et al., (2000), que encontró una prevalencia de uso de esteroides del $46 \%$ de la muestra afectada por DM. También son cercanos a la prevalencia establecida por parte de Pope et al. (1993), en la cual el $51 \%$ de los hombres de la muestra con DM usaba EAA.

Es necesario reconocer que este estudio tiene limitaciones en cuanto a las categorías del FFMI, que resultan de la abreviación de las clasificaciones establecidas por Kouri et al. (1995), Pope et al. (2000) y Kyle et al. (2003).

\section{Reconocimientos}

Este estudio fue parcialmente financiado por la Fundación MAPFRE (Artículo 83 - Contrato de Asistencia Técnica, UCTR08034).

\section{Conflicto de intereses}

Los autores declaran la inexistencia de conflicto de intereses.

\section{Referencias}

Barrientos, N., Escoto, C., Bosques, L. E., Enríquez, J. y Juárez, C. S. (2014). Interiorización de ideales estéticos y preocupación corporal en hombres y mujeres usuarios de gimnasio. Revista Mexicana de Trastornos Alimentarios, 5, 29-38.

Bhasin, S., Storer, T. W., Berman, N., Yarasheski, K. E., Clevenger, B., Phillips, K., ... Casaburi, R. (1997). Testosterone replacement increases fat-free mass and muscle size in hypogonodal men. Journal of Clinical Endocrinology Ẽ Metabolism, 82, 407-413. doi:10.1210/jc.82.2.407.

Bjørnebekk, A., Walhovd, K., Jørstad, M., Due-Tønnessen, P., Hullstein, I. y Fjell, A. (in press). Structural brain imaging of long term anabolic-androgenic steroid users and non-using weightlifters structural brain imaging of long term steroid use. Biological Psychiatry, 82, 294-302.

Cole, J. C., Smith, R, Halford, J. C. y Wagstaff, G. F. (2003). A preliminary investigation into the relationship between anabolic androgenic steroids use and the symptoms of reverse anorexia in both current and ex users. Psychopharmacology, 166, 424-429.

Coomber, R., Pavlidis, A., Hanley, G., Wilde, M., Schmidt, W. y Redshaw, C. (2014). The supply of steroids and other performance and image enhancing drugs (PIEDs) in one English city: fakes, counterfeits, supplier trust, common beliefs and access. Performance Enhancement $\mathcal{E}$ Health, 3, 135-144.

De la Torre, R. (1995). Esteroides anabolizantes-androgénicos [Anabolic-androgenic steroids]. En I. D. d. Drogodependencia (Ed.), Drogas y deporte: farmacología del doping, pp. 53-63.

González-Martí, I. (2012). Elementos que integran el perfil de la persona afectada por Dismorfia Muscular (Vigorexia). Prevalencia del trastorno en fisicoculturistas y usuarios de la sala de musculación. (Tesis doctoral inédita). Universidad de Castilla-La Mancha, Cuenca.

González-Martí, I., Fernández, J. G., Contreras, O. R. y Mayville, S. B. (2012). Validation of a Spanish version of the Muscle Appearance Satisfaction Scale: Escala de Satisfacción Muscular. Body Image, 9, 517-523. doi:10.1016/j. bodyim.2012.05.002.

González-Martí, I., Fernández, J. G. y Contreras, O. R. (2012). Contribución para el criterio diagnóstico de la Dismorfia Muscular (Vigorexia) [Contribution to the 
diagnostic criteria for Muscle Dysmorphia (Bigorexia)]. Revista de Psicología del Deporte, 21, 351-358.

González-Martí, I., Fernández, J. G., Hernández-Martínez, A. y Contreras, O. R. (2014). Physical perceptions and self-concept in athletes with muscle dysmorphia symptoms. The Spanish Journal of Psychology, 17 (e43), 1-7. doi:10.1017/sjp.2014.45.

González-Martí, I. Hernández-Martínez, A., Fernández, J. G. y Contreras, O. R. (2016). Prevalence of muscle dysmorphia on female weightlifters and bodybuilders. Artículo presentado para su publicación.

Gruber, A. J., Pope, H. G., Borowiecki, J. y Cohane, G. (1999). The development of the somatomorphic matrix: a biaxial instrument for measuring body image in men and women. En T. S. Olds, J. Dolloman y K. I. Norton (Eds.), Kinanthropometry VI (pp. 217-232). Sydney: International Society for the Advancement of Kinanthropometry.

Hildebrandt, T., Schlundt, D., Langenbucher, J. y Chung, T. (2006). Presence of muscle dysmorphia symptomology among male weightlifters. Comprehensive Psychiatry, 47, 127-135. doi:10.1016/j.comppsych.2005.06.001.

Hildebrandt, T., Lai, J., Langenbucher, J., Schneider, M., Yehuda, R. y Pfaff, D. (2011). The diagnostic dilemma of pathological appearance and performance enhancing drug use. Drug and Alcohol Dependence, 114, 1-11. doi:10.1016/j.drugalcdep.2010.09.018.

Hildebrandt, T., Harty, S. y Langenbucher, J. W. (2012). Fitness supplements as a gateway substance for anabolic-androgenic steroid use. Psychology of Addictive Behaviors, 26(4), 955-962. doi:10.1016/j.drugalcdep.2010.09.018.

Jackson, A. y Pollock, M. (1978). Generalized equations for predicting body density in man. British Journal Nutrition, 40, 497-504.

Jampel, J. D., Murray, S. B., Griffiths, S. y Blashill, A. J. (2016). Self-perceived weight and anabolic steroids misuse among US adolescent boys. Journal of Adolescent Health, 58, 397-402. doi:10.1016/j.jadohealth.2015.10.003.

Kanayama, G., Pope, H. G., Cohane, G. y Hudson, J. I. (2003). Risk factors for anabolic androgenic steroid use among weightlifters: a case-control study. Drug and Alcohol Dependence, 71, 77-86. doi:10.1016/S03768716(03)00069-3.

Kanayama, G., Barry, S., Hudson, J. I. y Pope Jr., H. G. (2006). Body image and attitudes toward male role in anabolic-androgenic steroid users. American Journal of Psychiatry, 163, 697-703. doi:10.1176/appi.ajp.163.4.697.

Kanayama, G., Hudson, J. I. y Pope Jr., H. G. (2009). Features of men with anabolic-androgenic steroid dependence: a comparison with nondependent AAS users and with AAS nonusers. Drug and Alcohol Dependence, 102, 1030-137. doi:10.1016/j.drugalcdep.2009.02.008.

Kouri, E. M., Pope, H. G., Katz, D. L. y Oliva, P. (1995). FatFree Mass Index in users and nonusers of anabolic-an- drogenic steroids. Clinical Journal of Sports Medicine, 5, 223-228. doi:10.1097/00042752-199510000-00003.

Kyle, U. G., Schutz, Y., Dupertuis, Y. M. y Pichard, C. (2003). Body composition interpretation: contributions of the fat-free mass index and the body fat mass index. Nutrition, 19, 597-604. doi:10.1016/S0899-9007(03)00061-3.

Murray, S. B., Griffiths, S., Mond, J. M., Kean, J. y Blashill, A. J. (En prensa). Anabolic steroid use and body image psychopathology in men: delineating between appearanceversus performance-driven motivations. Drug and Alcohol Dependence. doi:10.1016/j.drugalcdep.2016.06.008.

Olivardia, R., Pope, H. G. y Hudson, J. (2000). Muscle dysmorphia in male weightlifters: a case-control study. American Journal of Psychiatry, 157, 1291-1296. doi:10.1176/ appi.ajp.157.8.1291.

Pipet, S., Halpern, R., Woody, G. E. y Szobot, C. (2014). Association between AAS use, muscle dysmorphia and illicit drug use among gym frequenters. Drug and Alcohol Dependence, 140, e178. doi:10.1016/j.drugalcdep.2014.02.499.

Pope, H. G., Katz, D. \& Hudson, J. (1993). Anorexia nervosa and "reverse anorexia" among 108 male bodybuilders. Comprehensive Psychiatry, 34, 406-409.

Pope, H. G., Gruber, A., Choi, P., Olivardia, R. y Phillips, K. (1997). Muscle dysmorphia. An underrecognized form of Body Dysmorphic Disorder. Psychosomatics, 38, 548557. doi:10.1016/S0033-3182(97)71400-2.

Pope, H. G., Phillips, K. y Olivardia, R. (2000). The Adonis Complex. How to identify, treat and prevent body obsession in men and boys. New York: A Touchstone Book.

Pope, H. G., Kanayama, G., Ionescu-Pioggia, M. y Hudson, J. I. (2004). Anabolic steroid users' attitudes towards physicians. Addiction, 99, 1189-1194. doi:10.1111/j.13600443.2004.00781.x.

Pope, C. G., Pope, H. G., Menard, W., Fay, C., Olivardia, R. y Phillips, K. (2005). Clinical features of muscle dysmorphia among males with dysmorphic disorder. Body Image, 2, 395-399. doi:10.1016/j.bodyim.2005.09.001.

Pope, H. G., Kanayama, G. y Hudson, J. I. (2012). Risk for illicit anabolic-androgenic steroid use in male weightlifters: a cross-sectional cohort study. Biological Psychiatry, 71, 254-261. doi:10.1016/j.biopsych.2011.06.024.

Pope, H. G., Kanayama, G., Athey, A., Ryan, E., Hudson, J. I. y Baggish, A. (2014). The lifetime prevalence of anabolic-androgenic steroid use and dependence in Americans: current estimates. American Journal on Addiction 23, 371-373. doi:10.1111/j.1521-0391.2013.12118.x.

Rocha, M., Aguiar, F. y Ramos, H. (2014). O uso de esteroides androgénicos anabolizantes e outros suplementos ergogénicos - uma epidemia silenciosa. Revista Portuguesa de Endocrinologia, Diabetes e Metabolismo, 9, 98-105. doi:10.1016/j.rpedm.2014.09.002

Rodríguez-Testal, J. F., Senín-Calderon, C. y Perona-Garcelán, S. (2014). From DSM-IV-TR to DSM-5: Analysis of 
some changes. International Journal of Clinical and Health Psychology, 14, 221-231. doi:10.1016/j.ijchp.2014.05.002.

Rohman, L. (2009). The relationship between anabolic androgenic steroids and muscle dysmorphia: a review. Eating Disorder, 17, 187-199. doi:10.1080/10640260902848477.

Sokolova, M. V., González-Martí, I., Contreras, O. R. y Fernández, J. G. (2013). A case study of muscle dysmorphia disorder diagnostics. Expert Systems with Applications, 40, 4226-4231. doi:10.1016/j.eswa.2013.01.023.

Thiblin, I., Garmo, H., Garle, M., Holmberg, L., Byberg, L., Michaëlsson, K.,... Gedeborg, R. (2015). Anabolic steroids and cardiovascular risk: a national population-based cohort study. Drug and Alcohol Dependence, 152, 87-92. doi:10.1016/j.drugalcdep.2015.04.013.

Van Hout, M. C. y Kean, J. (2015). An exploratory study of image and performance enhancement drug use in a male British South Asian community. International Journal of Drug Policy, 26, 860-867. doi:10.1016/j.drugpo.2015.03.002. 